\title{
Rotation invariant subspaces of Besov and Triebel-Lizorkin space: compactness of embeddings, smoothness and decay of functions
}

\section{Leszek Skrzypczak}

\begin{abstract}
Let $H$ be a closed subgroup of the group of rotation of $\mathbb{R}^{n}$. The subspaces of distributions of Besov-Lizorkin-Triebel type invariant with respect to natural action of $H$ are investigated. We give sufficient and necessary conditions for the compactness of the Sobolev-type embeddings. It is also proved that $H$-invariance of function implies its decay properties at infinity as well as the better local smoothness. This extends the classical Strauss lemma. The main tool in our investigations is an adapted atomic decomposition.
\end{abstract}

\section{Introduction.}

The aim of the paper is to prove some assertions on the compactness of embeddings of some invariant subspaces of Besov-Lizorkin-Triebel classes into itself. We proved some decay properties of the invariant functions at infinity and near the origin. The local Hölder smoothness of the invariant functions is also investigated.

2000 Mathematics Subject Classification: 46E35, 42C15.

Keywords: Compact embeddings, Besov and Lizorkin-Triebel spaces, atomic decompositions. 
The phenomenon that symmetry conditions imply compactness of Sobolev embeddings has been observed by several authors. We refer to Berestycki and Lions [1], Coleman, Glazer, and Martin [5], Strauss [22], and Lions [17], where first order Sobolev spaces were regarded. The results are important for semi-linear elliptic equations. The case of the radial subspaces of the spaces of Besov-Lizorkin-Triebel type were investigated in [19], where the sufficient and necessary conditions for the compactness of the Sobolev-type embeddings are proved. In this paper we investigate the compactness of embeddings of subspaces invariant with respect to the action of a closed subgroup $H \subset O(n)$ and give sufficient and necessary conditions both for the function spaces and the subgroup.

Besov spaces $B_{p, q}^{s}\left(\mathbb{R}^{n}\right)$ and Lizorkin-Triebel spaces $F_{p, q}^{s}\left(\mathbb{R}^{n}\right)$ are natural generalizations of Sobolev and Hölder spaces, e.g. we have

$$
\begin{array}{lr}
F_{p, 2}^{0}\left(\mathbb{R}^{n}\right)=L_{p}\left(\mathbb{R}^{n}\right) & \text { (Lebesgue spaces) } \\
F_{p, 2}^{m}\left(\mathbb{R}^{n}\right)=W_{p}^{m}\left(\mathbb{R}^{n}\right) & (\text { Sobolev spaces) } \\
F_{p, 2}^{s}\left(\mathbb{R}^{n}\right)=H_{p}^{s}\left(\mathbb{R}^{n}\right) & \text { (Potential spaces) }
\end{array}
$$

if $1<p<\infty$ and

$$
B_{\infty, \infty}^{s}\left(\mathbb{R}^{n}\right)=\mathcal{C}^{s}\left(\mathbb{R}^{n}\right) \quad \text { (Hölder-Zygmund spaces) }
$$

if $s>0$. The standard references for the above function spaces are Frazier and Jawerth [9], [10], Peetre [18] or Triebel [23], [24].

A crucial part in the theory of embeddings of these spaces is taken by the number $s-n / p$ called the differential dimensions. If $0<p_{0} \leq p$ and if

$$
s_{0}-\frac{n}{p_{0}} \geq s-\frac{n}{p}
$$

then

$$
F_{p_{0}, q_{0}}^{s_{0}}\left(\mathbb{R}^{n}\right) \hookrightarrow F_{p, q}^{s}\left(\mathbb{R}^{n}\right), 0<q_{0}, q \leq \infty \text { (continuous embedding) }
$$

and

(3) $\quad B_{p_{0}, q_{0}}^{s_{0}}\left(\mathbb{R}^{n}\right) \hookrightarrow B_{p, q}^{s}\left(\mathbb{R}^{n}\right), 0<q_{0} \leq q \leq \infty$ (continuous embedding),

cf. Jawerth [14] or Sickel and Triebel [20]. We shall prove that if we restrict our interest to the subspaces $R_{H} B_{p, q}^{s}\left(\mathbb{R}^{n}\right)$ and $R_{H} F_{p, q}^{s}\left(\mathbb{R}^{n}\right)$ of functions (distributions) invariant with respect to the natural action of $H$ than 
most of the above embeddings become compact. In fact we are able to characterize all situations where those embeddings are compact.

For convenience we recall the definition of the function spaces. Let $\psi \in S\left(\mathbb{R}^{n}\right)$ be a function with $0 \leq \psi \leq 1$, supp $\psi \subset B(0,3 / 2)$ and $\psi(x)=1$ if $|x| \leq 1$. We put

$$
\begin{aligned}
& \varphi_{0}(x)=\psi(x), \\
& \varphi_{1}(x)=\psi\left(\frac{x}{2}\right)-\psi(x), \\
& \varphi_{j}(x)=\varphi_{1}\left(2^{-j+1} x\right), \quad j=2, \ldots
\end{aligned}
$$

The system $\left\{\varphi_{j}\right\}_{j=0}^{\infty}$ is the smooth resolution of unity and

$$
\begin{gathered}
\sum_{j=0}^{N} \varphi_{j}(x)=\psi\left(2^{-N} x\right), \\
\operatorname{supp} \varphi_{j} \subset\left\{x: 2^{j-1} \leq|x| \leq 3 \cdot 2^{j-1}\right\} .
\end{gathered}
$$

Let $\|\cdot\|_{p}$ denote the $L_{p}\left(\mathbb{R}^{n}\right)$ quasi-norm.

Definition 1. Let $s \in \mathbb{R}$ and $0<p, q \leq \infty$. Then

$$
B_{p, q}^{s}\left(\mathbb{R}^{n}\right)=\left\{f \in \mathcal{S}^{\prime}\left(\mathbb{R}^{n}\right):\left\|f \mid B_{p, q}^{s}\right\|=\left(\sum_{j=0}^{\infty} 2^{s j q}\left\|\mathcal{F}^{-1} \varphi_{j} \mathcal{F} f\right\|_{p}^{q}\right)^{1 / q}<\infty\right\} .
$$

If $0<p<\infty$ then

$$
\begin{aligned}
F_{p, q}^{s}\left(\mathbb{R}^{n}\right)=\left\{f \in \mathcal{S}^{\prime}\left(\mathbb{R}^{n}\right):\right. & \left\|f \mid F_{p, q}^{s}\right\| \\
& \left.=\left\|\left(\sum_{j=0}^{\infty} 2^{s j q}\left|\mathcal{F}^{-1} \varphi_{j} \mathcal{F} f(\cdot)\right|_{p}^{q}\right)^{1 / q}\right\|<\infty\right\} .
\end{aligned}
$$

Remark 1. The spaces are quasi-Banach spaces, Banach spaces if $p, q \geq 1$. The definition is independent of the chosen function $\psi$ up to the quasi-norm equivalence. The various equivalent characterizations can be found in [23], [24]. Sometimes we shall use the generic notation $A_{p, q}^{s}$ in place of $F_{p, q}^{s}$ and $B_{p, q}^{s}$, if we speak about the common properties of the above spaces.

Our main tool is an atomic decomposition so we recall also the atomic decomposition theorem. We follow [21] and [19], but the atomic decomposition theorem for the above spaces is due to Frazier and Jawerth, cf. [9], [10]. 
Definition 2. Let $s \in \mathbb{R}$ and $0<p \leq \infty$. Let $L$ and $M$ be integers such that $L \geq 0$ and $M \geq-1$. Let $B(x, r) \subset \mathbb{R}^{n}$ be a ball centered in $x$ with radius $r$.

a) A smooth function $a(x)$ is called an $1_{L}$-atom centered in $B(x, r)$ if there is a constant $C \geq 1$ such that

$$
\begin{aligned}
& \operatorname{supp} a \subset B(x, 2 r), \\
& \sup _{y \in \mathbb{R}^{n}}\left|D^{\alpha} a(y)\right| \leq 1, \quad|\alpha| \leq L .
\end{aligned}
$$

b) A smooth function $a(x)$ is called an $(s, p)_{L, M \text {-atom centered in }}$ $B(x, r)$ if

$$
\begin{aligned}
& \operatorname{supp} a \subset B(x, r) \text {, } \\
& \sup _{y \in \mathbb{R}^{n}}\left|D^{\alpha} a(y)\right| \leq r^{s-|\alpha|-n / p}, \quad|\alpha| \leq L, \\
& \left|\int_{\mathbb{R}^{n}} a(y) \varphi(y) d y\right| \leq r^{s+M+1+n / p^{\prime}}\left\|\varphi \mid C^{M+1}(\overline{B(x, 3 r)})\right\|, \\
& \varphi \in C^{\infty}\left(\mathbb{R}^{n}\right) \text {, where } 1 / p+1 / p^{\prime}=1 \text {. }
\end{aligned}
$$

Remark 2. If $M=-1$, then (11) becomes superfluous, it is covered by (10).

Remark 3. The following observations will be helpful from time to time. If $p_{0}<p_{1}$ and $s_{0}-n / p_{0}=s_{1}-n / p_{1}$, then an $\left(s_{0}, p_{0}\right)_{L, M}$-atom is also

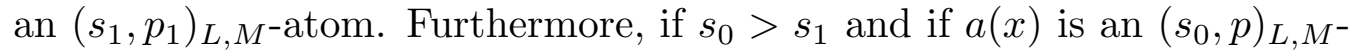
atom, then $r^{s_{0}-s_{1}} a(x)$ is an $\left(s_{1}, p\right)_{L, M}$-atom.

Let $j \in \mathbb{N}_{0}$ and $\left\{B\left(x_{j, i}, 2^{-j}\right)\right\}_{i \in \mathbb{N}_{0}}$ be a covering of $\mathbb{R}^{n}$ by balls such that the maximal number of the balls with non-empty intersection in this covering is finite. Such a covering is called uniformly locally finite and the maximal number is called the multiplicity of the covering. We may choose the sequence of the coverings $B_{j}=\left\{B\left(x_{j, i}, 2^{-j}\right)\right\}_{i \in \mathbb{N}_{0}}, j=0,1,2, \ldots$, with the same finite multiplicity. We recall the atomic decomposition theorem for Besov spaces. 
Theorem 1. Let $s \in \mathbb{R}$ and $0<p, q \leq \infty$. Let $L$ and $M$ be fixed integers satisfying the following conditions:

$$
\begin{aligned}
& L \geq([s]+1)_{+} \text {and } M \geq \max \left\{\left[-\sigma_{p}-s\right],-1\right\}, \\
& \sigma_{p}=n \max \left\{\frac{1}{p}-1,0\right\} .
\end{aligned}
$$

Let $B_{j}=\left\{B\left(x_{j, i}, 2^{-j}\right)\right\}_{i \in \mathbb{N}_{0}}, j=0,1, \ldots$, be a sequence of locally finite coverings with the same finite multiplicity.

a) Each $f \in B_{p, q}^{s}\left(\mathbb{R}^{n}\right)$ can be decomposed as follows

$$
f=\sum_{j=0}^{\infty} \sum_{i=0}^{\infty} s_{j, i} a_{j, i} \quad\left(\text { convergent in } \mathcal{S}^{\prime}\left(\mathbb{R}^{n}\right)\right)
$$

where $a_{0, i}$ is an s-atom related to the ball $B\left(x_{0, i}, r\right)$, and $a_{j, i}, j \neq 0$ is an $(s, p)$-atom related to the ball $B\left(x_{j, i}, r_{j}\right), s_{i}$ and $s_{j, i}$ are complex numbers with

$$
\left(\sum_{j=0}^{\infty}\left(\sum_{i=0}^{\infty}\left|s_{j, i}\right|^{p}\right)^{q / p}\right)^{1 / q}<\infty .
$$

( modification if either $p=\infty$ or $q=\infty$ ).

b) Conversely, suppose that $f \in \mathcal{S}^{\prime}\left(\mathbb{R}^{n}\right)$ can be represented as in (13) and (14). Then $f \in B_{p, q}^{s}\left(\mathbb{R}^{n}\right)$.

Furthermore, the infimum of (14) with respect to all admissible representations (for fixed sequence of coverings and fixed integers $L, M$ ) is an equivalent norm in $B_{p, q}^{s}\left(\mathbb{R}^{n}\right)$.

\section{Rotation invariant subspaces.}

Let $g$ be an isometry of $\mathbb{R}^{n}$. For $\varphi \in \mathcal{S}\left(\mathbb{R}^{n}\right)$ we put $\varphi^{g}(x)=\varphi(g x)$. If $f \in \mathcal{S}^{\prime}\left(\mathbb{R}^{n}\right)$ then $f^{g}$ is a tempered distribution defined by

$$
f^{g}(\varphi)=f\left(\varphi^{g^{-1}}\right), \quad \varphi \in \mathcal{S}\left(\mathbb{R}^{n}\right)
$$

where $g^{-1}$ denote the isometry inverse to $g$.

Let $O(n)$ denote a real orthogonal group on $n \times n$ matrices. The group $O(n)$ acts on $\mathbb{R}^{n}$ as a group of linear isometries. The group is a compact Lie transformation group. So its action on $\mathbb{R}^{n}$ is smooth. By $S O(n)$ we 
denote a real special orthogonal group that is the subgroup of $O(n)$ consists of matrices with determinant equal to 1 . It is a connected component of identity of $O(n)$.

Definition 3. Let $H$ be a subgroup of $O(n)$. We say that the tempered distribution $f$ is invariant with respect to $H$ if $f^{h}=f$ for any $h \in H$. For any possible $s, p, q$ we put

$$
\begin{aligned}
& R_{H} B_{p, q}^{s}\left(\mathbb{R}^{n}\right)=\left\{f \in B_{p, q}^{s}\left(\mathbb{R}^{n}\right): f \text { is invariant with respect to } H\right\} \\
& R_{H} F_{p, q}^{s}\left(\mathbb{R}^{n}\right)=\left\{f \in F_{p, q}^{s}\left(\mathbb{R}^{n}\right): f \text { is invariant with respect to } H\right\} .
\end{aligned}
$$

Remark 4. 1) The space $R_{H} A_{p, q}^{s}\left(\mathbb{R}^{n}\right)$ is a closed subspace of $A_{p, q}^{s}\left(\mathbb{R}^{n}\right)$. respectively. It follows immediately from the continuity of the linear operators $T_{h} f=f^{h}-f, h \in H$, in $A_{p, q}^{s}\left(\mathbb{R}^{n}\right)$ and the representation $R_{H} A_{p, q}^{s}\left(\mathbb{R}^{n}\right)=\bigcap_{h \in H} \operatorname{ker} T_{h}$. Thus they are quasi-Banach spaces with the quasi-norm induced from $A_{p, q}^{s}\left(\mathbb{R}^{n}\right)$. They are Banach spaces if $p \geq 1$ and $q \geq 1$.

2) The group $S O(n)$ acts transitively on the unit sphere $S^{n-1}=$ $\left\{x \in \mathbb{R}^{n}:|x|=1\right\}$ therefore the subspaces $R_{S O(n)} A_{p, q}^{s}\left(\mathbb{R}^{n}\right)$ consists of the radial distributions. We will denote this subspace by $R A_{p, q}^{s}\left(\mathbb{R}^{n}\right)$. If $H$ is a subgroup of $O(n)$ then $R_{S O(n)} A_{p, q}^{s}\left(\mathbb{R}^{n}\right) \subset R_{H} A_{p, q}^{s}\left(\mathbb{R}^{n}\right)$. Thus $R_{H} A_{p, q}^{s}\left(\mathbb{R}^{n}\right)=R A_{p, q}^{s}\left(\mathbb{R}^{n}\right)$ if $S O(n) \subset H$. These subspaces were investigated in [19].

3) Another example of rotation invariant subspaces are the subspaces corresponding to block radial (cylindrical) symmetries. Let $n=n_{1}+\cdots+$ $n_{m}$ with $n_{i} \geq 2$ and

$$
H=S O\left(n_{1}\right) \times \cdots \times S O\left(n_{m}\right)
$$

with the action of $h=\left(h_{1}, \ldots, h_{m}\right) \in H$ on $\mathbb{R}^{n}$ defined by

$$
h\left(x^{(1)}, \ldots, x^{(m)}\right)=\left(h_{1}\left(x^{(1)}\right), \ldots, h_{m}\left(x^{(m)}\right)\right), \quad x^{(i)} \in \mathbb{R}^{n_{i}} .
$$

The $H$ invariant subspaces of the first order Sobolev spaces were investigated in [17]. In particular the compactness of the Sobolev embeddings into $L_{p}$ spaces is proved.

4) We will assume that $H$ is a closed subgroup of $O(n)$. This assumption seems to be not very restrictive. The groups $S O(n)$ as well 
as $S O\left(n_{1}\right) \times \cdots \times S O\left(n_{m}\right)$ are of course closed subgroup of $O(n)$. Moreover, closed subgroup of real orthogonal groups $O(n)$ cover all compact Lie groups in that sense that any compact Lie group is isomorphic to a closed subgroup of certain orthogonal group $O(n)$, [2, Chapter 0, Theorem 5.1].

$5)$ We give another example of identification of the compact Lie group with the subgroup of orthogonal transformations that arise naturally in geometry. Let $X=G / K$ be a homogeneous Riemannian manifold with a Riemannian metric tensor $g$. Then $G$ is Lie group of isometries of $X$ and $K$ is a stability subgroup of a fixed point $o \in X$. The group $K$ is compact and acts naturally on the tangent space $T_{o} X$ by tangent mappings $d(k)_{o}, k \in$ $K$. The action is orthogonal on $T_{o} X$ since $g_{o}\left(d(k)_{o} v,(d k)_{o} w\right)=g_{o}(v, w)$, $v, w \in T_{o} X$. The nice exposition of the homogeneous manifolds can be find in [4, Chapter 3].

Proposition 1. Let $s \in \mathbb{R}$ and $1 \leq p, q \leq \infty$. If $H \subset O(n)$ is a closed subgroup then the space $R_{H} B_{p, q}^{s}\left(\mathbb{R}^{n}\right)\left(R_{H} F_{p, q}^{s}\left(\mathbb{R}^{n}\right)\right)$ is a complemented subspaces of $B_{p, q}^{s}\left(\mathbb{R}^{n}\right)\left(R_{H} F_{p, q}^{s}\left(\mathbb{R}^{n}\right)\right.$ respectively $)$.

Proof. We prove that there is a continuous projection $R: B_{p, q}^{s}\left(\mathbb{R}^{n}\right) \longrightarrow$ $R_{H} B_{p, q}^{s}\left(\mathbb{R}^{n}\right)$. The proof for $R_{H} F_{p, q}^{s}$ spaces is similar. The operator ( $I-$ $\Delta)^{m}, m \in \mathbb{N}$ commutes with all isometries of $\mathbb{R}^{n}$. So, it is an isomorphism of $B_{p, q}^{s}\left(\mathbb{R}^{n}\right)$ onto $B_{p, q}^{s-2 m}\left(\mathbb{R}^{n}\right)$ as well as an isomorphism of $R_{H} B_{p, q}^{s}\left(\mathbb{R}^{n}\right)$ onto $R_{H} B_{p, q}^{s-2 m}\left(\mathbb{R}^{n}\right)$, it is sufficient to regard $s>n / p$. In that case $B_{p, q}^{s}\left(\mathbb{R}^{n}\right) \subset C\left(\mathbb{R}^{n}\right)$.

If $n=1$ then we put $R f(x)=(f(x)+f(-x)) / 2$. It is clear that the operator is a continuous projection we are looking for.

If $n>1$ and the group $H$ is finite we put

$$
R f(x)=\frac{1}{|H|} \sum_{h \in H} f(h x) .
$$

The operator $R$ is once more a continuous projection.

Let $n>1$ and let $d h$ denotes the normalized Haar measure on $H$. We define

$$
R f(x)=\int_{H} f(h x) d x .
$$

The $H$-invariant functions are fixed points of $R$. We prove that $R$ is a bounded projection from $B_{p, q}^{s}\left(\mathbb{R}^{n}\right)$ onto $R_{H} B_{p, q}^{s}\left(\mathbb{R}^{n}\right)$.

The Fourier transform commutes with the orthogonal transformations of $\mathbb{R}^{n}$. So, $\mathcal{F}(f(g(\cdot)))(x)=\mathcal{F}(f)(g(x))$, if $g \in S O(n)$. In consequence

$$
\mathcal{F}(R f)=R(\mathcal{F}(f)),
$$


and

$$
\mathcal{F}^{-1} \varphi_{j} \mathcal{F}(R f)=R\left(\mathcal{F}^{-1} \varphi_{j} \mathcal{F} f\right)
$$

if the functions $\varphi_{j}$ are rotation invariant.

On the other hand, by the Minkowski inequality for integrals,

$$
\|R f\|_{p} \leq\|f\|_{p}
$$

So, the inequalities (15) and (16) imply

$$
\left\|R f\left|B_{p, q}^{s}\left(\mathbb{R}^{n}\right)\left\|=\left(\sum_{j=0}^{\infty} 2^{j s q}\left\|\mathcal{F}^{-1} \varphi_{j} \mathcal{F}(R f)\right\|_{p}^{q}\right)^{1 / q} \leq C\right\| f\right| B_{p, q}^{s}\left(\mathbb{R}^{n}\right)\right\|
$$

This finishes the proof.

\section{Compactness of the embeddings.}

In this section we give the sufficient and necessary condition for compactness of embeddings

$$
R_{H} A_{p_{0}, q_{0}}^{s_{0}}\left(\mathbb{R}^{n}\right) \hookrightarrow A_{p_{1}, q_{1}}^{s_{1}}\left(\mathbb{R}^{n}\right) .
$$

Our main tool will be $H$-invariant atomic decompositions of $B_{p, q}^{s}\left(\mathbb{R}^{n}\right)$. We start with notions of a separation and a discretization of $\mathbb{R}^{n}$ since they are useful for description of the atomic decomposition.

Definition 4. Let $\varepsilon>0$ be a positive number, $\alpha=1,2, \ldots$ be a positive integer and $X$ a nonempty subset of $\mathbb{R}^{n}$.

$A$ subset $\mathcal{H}$ of $X$ is said to be $\varepsilon$-separation of $X$, if the distance between any two distinct points of $\mathcal{H}$ is greater than or equal to $\varepsilon$.

$A$ subset $\mathcal{H}$ of $X$ is called an $(\varepsilon, \alpha)$-discretization of $X$ if it is an $\varepsilon$-separation of $X$ and

$$
X \subset \bigcup_{x \in \mathcal{H}} B(x, \alpha \varepsilon)
$$

Remark 5. 1) Any $\varepsilon$-separation is a finite or countable subset of $\mathbb{R}^{n}$.

2) For the notation of $\varepsilon$-separations and discretization as well as their importance in geometry we confer to [3, Chapter 4]. Please note, that our notion of discretization is a bit different to that one in Chavel's book. 
We denote by $|X|$ cardinality of a set $X$.

Lemma 1. Let $\varepsilon>0$ and $\alpha \in \mathbb{N}$.

a) For any nonempty subset $X$ of $\mathbb{R}^{n}$ there is a $(\varepsilon, 1)$-discretization of $X$.

b) Let $m$ be a positive integer. If $\mathcal{H}$ is an $(\varepsilon, \alpha)$-discretization of $\mathbb{R}^{n}$ and $m \geq \alpha$ then the family $\{B(x, m \varepsilon)\}_{x \in \mathcal{H}}$ is an uniformly locally finite covering of $\mathbb{R}^{n}$ with multiplicity that can be estimates from above by constant depending on $n$ and $m$, but independent of $\varepsilon$.

Proof. The proof is standard. It follows from the Zorn lemma that for any nonempty set $X$ there exist the maximal $\varepsilon$-separation $\mathcal{H}$ of $X$. But then

$$
X \subset \bigcup_{x \in \mathcal{H}} B(x, \varepsilon) .
$$

by maximality of $\mathcal{H}$. This proves a).

We prove b). If $x_{i}, x_{j} \in \mathcal{H}$ and $x_{i} \neq x_{j}$ then

$$
B\left(x_{i}, \frac{\varepsilon}{2}\right) \cap B\left(x_{j}, \frac{\varepsilon}{2}\right)=\varnothing .
$$

Let $y \in \mathbb{R}^{n}$ and $I(y)=\{x \in \mathcal{H}: y \in B(x, m \varepsilon)\}$. Then by (17) we get

$$
\begin{aligned}
c_{n}|I(y)|\left(\frac{\varepsilon}{2}\right)^{n} & \leq \operatorname{vol}\left(\bigcup_{x \in I(y)} B\left(x, \frac{\varepsilon}{2}\right)\right) \\
& \leq \operatorname{vol}(B(y,(m+1) \varepsilon) \\
& \leq c_{n}(m+1)^{n} \varepsilon^{n} .
\end{aligned}
$$

Thus the multiplicity of the covering is less or equaled to $C_{n}(m+1)^{n}$.

Let $H \subset O(n)$ be a closed subgroup of $O(n)$ and $\mathcal{H}$ any $\varepsilon$-separation of $\mathbb{R}^{n}$. For any $x \in \mathcal{H}$ we put

$$
\mathcal{H}(x, H)=\{y \in \mathcal{H}: \text { exists } h \in H, h \cdot x=y\} .
$$

Definition 5. Let $H$ be a closed subgroup of $O(n)$. Let $\mathcal{H}$ be a $(\varepsilon, \alpha)$ discretization of $\mathbb{R}^{n}$. We say that the discretization $\mathcal{H}$ is $H$-rich if there are constants $c>0$ and $b \geq 1$ such that for any $x \in \mathcal{H}, x \neq 0$ the following estimate

$$
c\left(|x| \varepsilon^{-1}\right)^{b} \leq|\mathcal{H}(x, H)|
$$

holds. 
Remark 6. Since 0 is a fixed point of $H$ the set $\mathcal{H}(x, H)$ is contained in a sphere $S^{n-1}(0,|x|)$. This implies the following upper bound of the cardinality of $\mathcal{H}(x, H)$

$$
|\mathcal{H}(x, H)| \leq C\left(\varepsilon^{-1}|x|\right)^{n-1} .
$$

Moreover $H \cdot x, x \neq 0$, is a smooth submanifold of $\mathbb{R}^{n}$, therefore one can take

$$
b=\min _{x \in S^{n-1}} \operatorname{dim} H \cdot x .
$$

This value of $b$ is optimal.

Lemma 2. Let $H$ be a closed subgroup of $O(n)$. Then the following conditions are equivalent:

1) For any $x \neq 0$ there orbit $H \cdot x=\{h \cdot x: h \in H\}$ is infinite.

2) For any $x \in S^{n-1}$ there orbit $H \cdot x=\{h \cdot x: h \in H\}$ is infinite.

3) For any $j \in \mathbb{N}_{0}$ and there exist a $\left(2^{-j}, \alpha\right)$-discretization $\mathcal{H}_{j}$ of $\mathbb{R}^{n}$ such that:

- the constant $\alpha$ is independent of $j$,

- the discretization $\mathcal{H}_{j}$ is $H$-rich,

- the constants $(c, b)$ in (18) are independent of $j$.

Proof. Step 1. The equivalence 1) if and only if 2) is obvious. The group $H$ is a closed subgroup of a compact group therefore it is compact and it has a finite many connection components. Let $H_{0}$ denote a connection component of the identity of $H$. It is a closed subgroup of $H$.

The group $H$ is a closed subgroup of the compact Lie group $O(n)$ therefore it is a Lie subgroup of $O(n)$ if equipped with the unique differential structure, $c f$. [12, Chapter 2, Theorem 2.3]. In consequence it acts analytically of the unit sphere $S^{n-1}$ as a group of isometries. Any orbit $H \cdot x$ is a regular submanifold of $S^{n-1}$ of dimension $k_{x}=\operatorname{dim} H-\operatorname{dim} H_{x}$, where $H_{x}$ is a stability subgroup of $x$ in $H$, cf. [2, Chapter 6, Corollary 1.3]. So if the orbit $H \cdot x$ is infinite then it is a regular submanifolds of dimension $k_{x} \geq 1$.

The group $H$ acts on the family of its connected components by translations. In consequences if $T_{h}: H_{0} \longrightarrow H_{1}$ is a translations that defines an diffeomorphism of $H_{0}$ onto a connected component $H_{1}$ then it defines also a diffeomorphism of an orbit $H_{0} \cdot x$ onto $H_{1} \cdot x$. In consequence the 
orbit $H \cdot x$ is infinite if and only if infinite is the orbit $H_{0} \cdot x$. So, $H_{0} \cdot x$ is a regular connected submanifolds of dimension $k_{x} \geq 1$.

Step 2. We prove that 2) implies 3 ). First we notice that there is a positive constant $\delta$ such that for any $x \in S^{n-1}$ we have

$$
H_{0} \cdot x \nsubseteq B(x, \delta)
$$

Indeed, if the last statement is not true then for any $m \in \mathbb{N}$ there exist $x_{m} \in S^{n-1}$ such that $H_{0} \cdot x_{m} \subseteq B(x, 1 / m)$. But $S^{n-1}$ is a compact set therefore the sequence $\left\{x_{m}\right\}_{m \in \mathbb{N}}$ has got a convergent subsequence. To simplify the notation we assume that $x_{m} \longrightarrow y$, if $m \longrightarrow \infty$. Let us choose $\varepsilon>0$ then there is $x_{m}$ such that $1 / m<\varepsilon / 3$ and $\left|y-x_{m}\right| \leq \varepsilon / 3$. So, for any $h \in H_{0}$ we have $|h \cdot y-y| \leq \varepsilon$. Thus, $H_{0} \cdot y \subset B(y, \varepsilon)$ for any $\varepsilon$. Then $H_{0} \cdot y=\{y\}$, but this is impossible since any $H_{0}$-orbit is infinite, cf. Step 1.

Let us choose $\varepsilon, 0<\varepsilon \leq 1$. Let $x_{\varepsilon, 1}, \ldots, x_{\varepsilon, k} \in S^{n-1}$ be a maximal set such that:

1) $x_{\varepsilon, 1} \in S^{n-1}$,

2) $x_{\varepsilon, i} \in S^{n-1}$ and $\operatorname{dist}\left(x_{i}, \bigcup_{l=1}^{i-1} H_{0} \cdot x_{\varepsilon, l}\right)=\varepsilon$, for $i=2, \ldots k$.

Since any orbit is a regular submanifold of $S^{n-1}$ and $S^{n-1}$ is compact such set is finite. In particular if $H_{0}$ acts transitively on the sphere then the set has only one element.

For any orbit $H_{0} \cdot x_{\varepsilon, i}$ there is an $(\varepsilon, 1)$-discretization of $H_{0} \cdot x_{\varepsilon, i}$. We will denote this discretization by $X_{\varepsilon, i}$. The set $X_{\varepsilon}=\bigcup_{i=1}^{k} X_{\varepsilon, i}$ is then the $(\varepsilon, 2)$ - discretization of $S^{n-1}$. The orbit $H_{0} \cdot x_{i}$ is a connected regular submanifold of $S^{n-1}$ of dimension $k_{i} \geq 1$ therefore (21) implies, that the inequalities

$$
\left|X_{\varepsilon, i}\right| \geq \begin{cases}1, & \text { if } \delta<\varepsilon, \\ \left\lfloor\frac{\delta}{\varepsilon}\right\rfloor, & \text { if } \delta \geq \varepsilon,\end{cases}
$$

holds for any discretization $I_{\varepsilon, i}$. Here $\lfloor t\rfloor$ denotes the integer part of $t \in \mathbb{R}_{+}$.

Let us choose $j \in \mathbb{N}_{0}$. We construct the $\left(2^{-j}, 2\right)$-discretization $\mathcal{H}_{j}$ of $\mathbb{R}^{n}$ that is $H$-rich. We assume that the set $\mathcal{H}_{j}$ consists of 0 and all the points satisfying the following conditions:

$$
\begin{gathered}
|y|=N 2^{-j}, \quad N=1,2, \ldots, \\
\text { exists } X_{N^{-1}, i}, \quad|y|^{-1} y \in X_{N^{-1}, i} .
\end{gathered}
$$


It should be obvious that $\mathcal{H}_{j}$ is an $\left(2^{-j}, 3\right)$-discretization of $\mathbb{R}^{n}$. Moreover by (22) and (24) we have

$$
\frac{\delta}{2} 2^{j}|y| \leq\left|X_{N^{-1}, i}\right|=\left|\mathcal{H}_{j}(y)\right|
$$

for any $0 \neq y \in \mathcal{H}_{j}$.

Step 3. It remains to prove that 3) implies 2). Let $x \in S^{n-1}$. We put $x_{N}=N \cdot x$. There exists $y_{N} \in \mathcal{H}_{0}$ such that $x_{N} \in B\left(y_{N}, \alpha\right)$. Let $M$ denotes the multiplicity of the covering $\{B(y, \alpha)\}_{y \in \mathcal{H}_{0}}$, cf. Lemma 1. We have

$$
|H \cdot x|=\left|H \cdot x_{N}\right| \geq M^{-1}\left|H \cdot y_{N}\right| \geq c M^{-1} N^{b}
$$

since $H$ is a group of isometries of $\mathbb{R}^{n}$. Thus $|H \cdot x|=\infty$. This finishes the proof.

Remark 7. 1) The simplest example of a group satisfying the assumption of the above Lemma is $S O(n)$. In this case $b=n-1$. The theory of corresponding radial subspaces is elaborated in [19].

2) Another example are block radial (cylindrical) symmetries regarded in Remark 4. In this case we can give more explicit construction of the $H$ rich discretizations. Let $\left\{x_{k, l}^{(i)}\right\}, k \in \mathbb{N}_{0}$ and $l=0, \ldots, k^{n_{i}-1}$, be a $\left(2^{-j}, \alpha_{i}\right)$ discretization in $\mathbb{R}^{n_{i}}$ that is $S O\left(n_{i}\right)$-rich, $c f$. [19] for the construction. We put

$$
\mathcal{H}_{j}=\left\{x_{\widetilde{k}, \tilde{l}}=\left(x_{k_{1}, l_{1}}^{(1)}, \ldots, x_{k_{m}, l_{m}}^{(m)}\right): k_{i} \in \mathbb{N}_{0}, l_{i}=0, \ldots, k_{i}^{n_{i}-1}\right\}
$$

and

$$
\alpha=\sqrt{n} \max _{i}\left\{\alpha_{i}\right\}
$$

The set $\mathcal{H}_{j}$ is a $\left(2^{-j}, \alpha\right)$-discretization of $\mathbb{R}^{n}$ Let $x_{\widetilde{k}, \widetilde{l}} \in \mathcal{H}_{j}$ with $\widetilde{k} \neq 0$. Then

$$
0<2^{-j} \max _{i} k_{i} \leq|x| \leq \sqrt{n} 2^{-j} \max _{i} k_{i}
$$

So

$$
\left|\mathcal{H}_{j}\left(x_{\widetilde{k}, \tilde{l}}, H\right)\right| \geq k_{1}^{n_{1}-1} \ldots k_{m}^{n_{m}-1} \geq \max \left\{k_{1}^{n_{1}-1}, \ldots, k_{m}^{n_{m}-1}\right\} \geq c\left(2^{j}|x|\right)^{b}
$$

with $b=\min \left\{n_{1}, \ldots, n_{m}\right\}-1$. 
3) Let $n=n_{1}+\cdots+n_{m}$ and let

$$
H=K_{1} \times \cdots \times K_{m}
$$

be a subgroup of $O(n)$ with $K_{i} \subset O\left(n_{i}\right)$. The action of $h=\left(h_{1}, \ldots, h_{m}\right) \in$ $H$ on $\mathbb{R}^{n}$ is defined in the same way as above. The group $H$ satisfies the assumptions of the Lemma 2 if and only if every group $K_{i}$ satisfies the assumptions of the Lemma.

4) To give another example we continue the last point of Remark 4 . Let $X=G / K$ be a Riemannian symmetric space of compact or noncompact type. In that case the group $G$ is semi-simple. The rank $r(X)$ of the symmetric spaces is a maximal dimension of a flat totally geodesic submanifold of $X$. The group $K$ acts transitively on the sphere centered at the origin $o \in X$ of $X$ if and only if $r(X)=1$. For any $x \in X$ the exist a flat totally geodesic submanifold trough $x$ and $o$ of dimension $r(X)$. Moreover, maximal flat totally geodesic submanifolds trough the origin $o \in X$ are in one to one correspondence with maximal abelian subspaces of the Iwasawa decomposition of the Lie algebra $\mathfrak{g}$ of $G$. The group $\operatorname{Ad}_{G}(K)$ acts transitively on the family the maximal abelian subspaces. So, if the $r(X)>1$ then $\operatorname{Ad}_{G}(K)$ do not act transitively on the sphere but any orbit is infinite. The best reference for geometry of symmetric space in Helgason's book [12]. For the analysis on tangent spaces of symmetric spaces including the harmonic analysis of $\operatorname{Ad}_{G}(K)$ invariant functions we refer to [13, Chapter 3, sections 7 and 8].

5) Let

$$
m=\frac{(n-1)(n+2)}{2}, \quad n>1 \text {. }
$$

We describe more precisely the action of $S O(n)$ onto $\mathbb{R}^{m}$ connected with the decomposition of a space $S L(n, \mathbb{R}) / S O(n)$, that is a symmetric spaces of rank $n-1$. We identify $\mathbb{R}^{m+1}$ with a space $\mathfrak{s}$ of $n \times n$ real symmetric matrices and $\mathbb{R}^{m}$ with a subspace $\mathfrak{p}$ of $\mathfrak{s}$ of matrices with zero trace. We define the scalar product in $\mathfrak{s}$ by

$$
\mathcal{K}(A, C)=2 n \operatorname{tr}(A C), \quad(\text { the Killing for of } S L(n, \mathbb{R}))
$$

Equipped with the above scalar product $\mathfrak{s}$ is an euclidean space and $\mathfrak{p}$ an euclidean subspace of codimension 1 . We define the action of $S O(n)$ onto $\mathfrak{p}$ by

$$
\tau(B): \mathfrak{p} \ni A \longmapsto B^{-1} A B \in \mathfrak{p}, \quad B \in S O(n) .
$$


It follows by the elementary properties of the trace and transpose matrices that $B^{-1} A B \in \mathfrak{p}$ if $A \in \mathfrak{p}$, and that $\mathcal{K}(\tau(A), \tau(C))=\mathcal{K}(A, C)$. The spaces $\mathfrak{a}$ of diagonal matrices in $\mathfrak{p}$ is an $n-1$ dimensional linear subspace. Any symmetric matrix is diagonalizable so for any $A \in \mathfrak{p}$ there exists $B \in S O(n)$ and $C \in \mathfrak{a}$ such that $A=B^{-1} C B$. Thus

$$
\mathfrak{p}=\bigcup_{B \in S O(n)} \tau(B)(\mathfrak{a}) \text {. }
$$

An easy calculation shows that if both matrices $A$ and $B^{-1} A B$ are diagonal then they differ only by permutation of the elements on the diagonal. So $S O(n)$ acts on any subspace of the form $\tau(B)(\mathfrak{a})$ as a group of permutation of $n$ elements.

So any system of real numbers $\lambda_{1}, \ldots, \lambda_{n-1}$ appoints exactly one orbit of the group $S O(n)$ in $\mathfrak{p}$. The orbit consists of symmetric matrices of eigenvalues $\lambda_{1}, \ldots, \lambda_{n-1}$ and $\lambda_{n}=-\lambda_{1}-\cdots-\lambda_{n-1}$. If the system contains at least one number no equaled to 0 then the orbit is infinite. But if $n>2$ then $S O(n)$ does not act transitively on any sphere centered at the origin in $\mathfrak{a}$, hence also in $\mathfrak{p}$.

Theorem 2. Let $H \subset O(n)$ be a closed subgroup of $O(n), n \geq 2$. Let $R_{H} A_{p, q}^{s}\left(\mathbb{R}^{n}\right)$ be an $H$-invariant subspace of $A_{p, q}^{s}\left(\mathbb{R}^{n}\right)$. The embedding

$$
R_{H} A_{p_{0}, q_{0}}^{s_{0}}\left(\mathbb{R}^{n}\right) \hookrightarrow R_{H} A_{p_{1}, q_{1}}^{s_{1}}\left(\mathbb{R}^{n}\right)
$$

is compact if and only if,

i) for any $x \in S^{n-1}$ the orbit $H \cdot x$ is infinite,

ii) $p_{0}<p_{1}$ and $s_{0}-n / p_{0}>s_{1}-n / p_{1}$.

Remark 8. 1) If $n=1$ then the only invariant subspaces is a subspaces of even distributions. In that case the embeddings are not compact. This was proved in [19].

2) The compactness of embeddings of invariant subspaces of order one Sobolev spaces, in case of the block radial symmetries was regarded in [17].

Proof. Step 1. Preparations. In view of the elementary embeddings

$$
R_{H} B_{p, \min \{p, q\}}^{s} \subset R_{H} F_{p, q}^{s} \subset R_{H} B_{p, \max \{p, q\}}^{s},
$$

$c f .$, e.g. $[23,2.3 .2]$, it is enough to deal with the subspaces of the Besov spaces. Let $m$ be a natural number. The operator $(\mathrm{id}-\Delta)^{m}$ maps a Besov 
space $B_{p, q}^{s}\left(\mathbb{R}^{n}\right)$ isomorphically onto $B_{p, q}^{s-m}\left(\mathbb{R}^{n}\right)$ and it is invariant with respect to any isometry of $\mathbb{R}^{n}$ so it maps also $R_{H} B_{p, q}^{s}\left(\mathbb{R}^{n}\right)$ isomorphically onto $R_{H} B_{p, q}^{s-m}\left(\mathbb{R}^{n}\right)$. Consequently, the question whether an embedding like $R B_{p_{0}, q_{0}}^{s_{0}}\left(\mathbb{R}^{n}\right) \hookrightarrow R B_{p_{1}, q_{1}}^{s_{1}}\left(\mathbb{R}^{n}\right)$ is compact can be lifted to arbitrary large smoothness $s_{0}$ and $s_{1}$ by employing the properties of $\left((\mathrm{id}-\Delta)^{m}\right)^{-1}$. So we may assume $s_{0}>\sigma_{p_{0}}$ and $s_{1}>\sigma_{p_{1}}$, cf. (12).

Step 2. In this step we prove the sufficiency of the conditions i) and ii). Substep 2.1. By Lemma 2 there is a positive integer $\alpha$ such that for any $j \in \mathbb{N}_{0}$ there exist a $\left(2^{-j}, \alpha\right)$-discretization $\mathcal{H}_{j}$ of $\mathbb{R}^{n}$ such that the following inequalities hold

$$
c\left(|x| 2^{j}\right)^{b} \leq\left|\mathcal{H}_{j}(x, H)\right|,
$$

where the constants $c>0$ and $b \geq 1$ are independent of $j$. It follows from Lemma 1 that a family of balls $\left\{B\left(x, \beta 2^{-j}\right)\right\}_{x \in \mathcal{H}_{j}}, \beta \geq \alpha$ forms a uniformly locally finite covering of $\mathbb{R}^{n}$. Moreover, the multiplicities of the coverings can be bounded from above by the constant independent of $j$. Thus the above coverings are suitable for atomic decompositions.

We put

$$
\begin{aligned}
\mathcal{H}_{j}=\left\{x_{j, k, m, \ell} \in \mathbb{R}^{n}:\right. & k=0,1,2 \ldots, \\
m & =0, \ldots, c(j, k), \ell=0,1, \ldots, C(j, k, m)\}
\end{aligned}
$$

and assume that $x_{j, 0,0,0}=0$ and

$$
(k-1) 2^{-j}<\left|x_{j, k, m, \ell}\right| \leq k 2^{-j}
$$

and

$$
x_{j, k, m, \ell} \in \mathcal{H}_{j}\left(x_{j, k, m, 0}, H\right)
$$

if $k=1,2, \ldots$ It follows from (19) and (25) that

$$
c k^{b} \leq C(j, k, m) \leq C k^{n-1} .
$$

On the other hand since the volume of the ring $\left\{x:(k-1) 2^{-j} \leq|x| \leq\right.$ $\left.k 2^{-j}\right\}$ is equivalent to $k^{n-1} 2^{-j n}$ and $\mathcal{H}_{j}$ is the $2^{-j}$-separation therefore

$$
\sum_{m=0}^{c(j, k)} C(j, k, m) \leq C k^{n-1}
$$

In consequence

$$
1 \leq c(j, k) \leq C k^{n-1-b}
$$


Substep 2.2. We describe the atomic decomposition that is suitable to our purpose. We can be brief here because it is similar to that one given in [19] for radial case, which is parallel to the construction given in [9].

The starting point is a Calderon type formula. As usual $\mathcal{F}$ denotes the Fourier transform and $\mathcal{F}^{-1}$ its inverse. Let $\Theta_{0}$ and $\Theta$ be radial Schwartz functions satisfying

$$
\left|\mathcal{F} \Theta_{0}(\xi)\right|>0, \quad \text { if }|\xi| \leq 2
$$

and

$$
|\mathcal{F} \Theta(\xi)|>0, \quad \text { if } \quad \frac{1}{2} \leq|\xi| \leq 2 .
$$

Then there exists two further radial Schwartz functions $\varphi_{0}, \varphi$ and a positive number $\delta$ such that

$$
\begin{aligned}
& \operatorname{supp} \varphi_{0} \subset\{\xi:|\xi| \leq 2\}, \quad\left|\varphi_{0}(\xi)\right|>0, \quad \text { if }|\xi| \leq \delta, \\
& \operatorname{supp} \varphi \subset\left\{\xi: \frac{1}{2} \leq|\xi| \leq 2\right\}, \quad|\varphi(\xi)|>0, \quad \text { if } \frac{3}{5} \leq|\xi| \leq \frac{5}{3}
\end{aligned}
$$

and

$$
\mathcal{F} \Theta_{0}(\xi) \varphi_{0}(\xi)+\sum_{j=1}^{\infty} \mathcal{F} \Theta\left(2^{-j} \xi\right) \varphi\left(2^{-j} \xi\right)=1,
$$

for all $\xi \in \mathbb{R}^{n}$, cf. e.g. [9]. The above identity gives

$$
\begin{aligned}
f(x)= & \int \Theta_{0}(x-y) \mathcal{F}^{-1}\left(\varphi_{0}(\xi) \mathcal{F} f(\xi)\right)(y) d y \\
& +\sum_{j=1}^{\infty} \int 2^{j n} \Theta\left(2^{j}(x-y)\right) \mathcal{F}^{-1}\left(\varphi\left(2^{-j} \xi\right) \mathcal{F} f(\xi)\right)(y) d y
\end{aligned}
$$

for all tempered distributions $f$ (convergence in $\mathcal{S}^{\prime}\left(\mathbb{R}^{n}\right)$ ). We may choose $\Theta_{0}$ and $\Theta$ such that

$$
\operatorname{supp} \Theta_{0} \subset\left\{\xi:|\xi| \leq \frac{1}{2}\right\}, \quad \operatorname{supp} \Theta \subset\left\{\xi:|\xi| \leq \frac{1}{2}\right\} .
$$

Using the abbreviations

$$
f_{j}(x)= \begin{cases}\mathcal{F}^{-1}\left(\varphi_{0}(\xi) \mathcal{F} f(\xi)\right)(x), & \text { if } j=0 \\ \mathcal{F}^{-1}\left(\varphi\left(2^{-j} \xi\right) \mathcal{F} f(\xi)\right)(x), & \text { if } j>0\end{cases}
$$


we define

$$
s_{j, k, m, \ell}= \begin{cases}D_{1} \sup _{y \in B\left(x_{0, k, m, \ell}, \alpha\right)}\left|f_{0}(y)\right| & \text { if } j=0, \\ D_{2} 2^{j(s-n / p)} \sup _{y \in B\left(x_{j, k, m, \ell}, \alpha 2^{-j}\right)}\left|f_{j}(y)\right|, & \text { if } j>0,\end{cases}
$$

and

$$
\begin{aligned}
& a_{j, m, k, \ell}(x) \\
& = \begin{cases}\frac{1}{s_{0, \ell}} \int_{B\left(x_{0, k, m, \ell}, \alpha\right)} \Theta_{0}(x-y) f_{0}(y) d y, & \text { if } j=0, \\
\frac{2^{j n}}{s_{j, \ell}} \int_{B\left(x_{j, k, m, \ell}, \alpha 2^{-j}\right)} \Theta\left(2^{j}(x-y)\right) f_{j}(y) d y, & \text { if } j>0,\end{cases}
\end{aligned}
$$

where $D_{1}, D_{2}>0$ are normalization constants. The above formulae lead to the following atomic decompositions

$$
f(x)=\sum_{j=0}^{\infty} \sum_{k, m, \ell} s_{j, k, m, \ell} a_{j, k, m, \ell}(x)
$$

(convergence in $\mathcal{S}^{\prime}\left(\mathbb{R}^{n}\right)$ ), and

$$
\left(\sum_{j=0}^{\infty}\left(\sum_{k, m, \ell}\left|s_{j, k, m, \ell}\right|^{p}\right)^{q / p}\right)^{1 / q} \leq C\left\|f \mid B_{p, q}^{s}\left(\mathbb{R}^{n}\right)\right\|
$$

with some constant $C$ independent of $f$. Since $s>\sigma_{p}$ no moment condition for atoms is needed.

If $f \in R_{H} B_{p, q}^{s}\left(\mathbb{R}^{n}\right)$ then the functions $f_{j}$ defined in (28) are also $H$-invariant. Thus the properties of the discretizations $\mathcal{H}_{j}$ described in Substep 2.1 and (30) imply $s_{j, k, m, \ell}=s_{j, k, m, 0}$ if $\ell=0, \ldots, C(j, k, m)$. So we simplify the notation putting

$$
s_{j, k, m}=s_{j, k, m, \ell}, \quad \ell=0, \ldots, C(j, k, m) .
$$

Substep 2.3. It is known that the above embeddings are continuous, $c f$., e.g. Jawerth [14] or Triebel [23, 2.7.1]. by

First we assume $p_{0}<p_{1}<\infty$. Suppose $f \in R_{H} B_{p_{0}, q_{0}}^{s_{0}}\left(\mathbb{R}^{n}\right)$ is given

$$
f(x)=\sum_{j=0}^{\infty} s_{j, 0,0} a_{j, 0,0,0}(x)+\sum_{j=0}^{\infty} \sum_{k=0}^{\infty} \sum_{m, \ell} s_{j, k, m} a_{j, k, m, \ell}(x)
$$


Since $s_{0}-n / p_{0}>s_{1}-n / p_{1}$ the above decomposition is an $\left(s_{0}, p_{0}\right)$ as well as an $\left(s_{1}, p_{1}\right)$ decomposition. Without loss of generality we may assume $q_{1}=q_{0}=\infty$. Let $E$ be a bounded set in $R B_{p_{0}, \infty}^{s_{0}}\left(\mathbb{R}^{n}\right)$. Then there is a general constant $C$ such that for any $f \in E$ we have

$$
\sup _{j=0,1, \ldots}\left|s_{j, 0,0}\right|+\sup _{j=0,1, \ldots}\left(\sum_{k=1}^{\infty} \sum_{m=0}^{c(j, k)} C(j, k, m)\left|s_{j, k, m}\right|^{p_{0}}\right)^{1 / p_{0}}<C,
$$

cf. (32). The monotonicity of the sequence spaces $l_{p}(\mathbb{N})$ implies

$$
\sup _{j=0,1, \ldots}\left|s_{j, 0,0}\right|+\sup _{j=0,1, \ldots}\left(\sum_{k=1}^{\infty} \sum_{m=0}^{c(j, k)} C(j, k, m)^{p_{1} / p_{0}}\left|s_{j, k, m}\right|^{p_{1}}\right)^{1 / p_{1}}<C .
$$

The last inequality and (26) implies

$$
\sup _{j=0,1, \ldots}\left|s_{j, 0,0}\right|+\sup _{j=0,1, \ldots}\left(\sum_{k=1}^{\infty} k^{\sigma} \sum_{m=0}^{c(j, k)} C(j, k, m)\left|s_{j, k, m}\right|^{p_{1}}\right)^{1 / p_{1}}<C,
$$

where $\sigma=b\left(p_{1} / p_{0}-1\right)>0$.

Let us fix $\varepsilon>0$. Let $N>(C / \varepsilon)^{p_{1} / \sigma}$, where $C$ is as above. From (35) it follows

$$
\begin{aligned}
\sup _{j=0,1, \ldots} & \left(\sum_{k=N}^{\infty} \sum_{m=0}^{c(j, k)} C(j, k, m)\left|s_{j, k, m}\right|^{p_{1}}\right)^{1 / p_{1}} \\
& \leq N^{-\sigma / p_{1}} \sup _{j=0,1, \ldots}\left(\sum_{k=N}^{\infty} k^{\sigma} \sum_{m=0}^{c(j, k)} C(j, k, m)\left|s_{j, k, m}\right|^{p_{1}}\right)^{1 / p_{1}} \\
& <\varepsilon .
\end{aligned}
$$

For given $f$ we put

$$
\begin{gathered}
f_{1}(x)=\sum_{j=0}^{\infty} s_{j, 0,0} a_{j, 0,0,0}(x)+\sum_{j=0}^{\infty} \sum_{k=1}^{N-1} \sum_{m=1}^{c(j, k)} \sum_{\ell=1}^{C(j, k, m)} s_{j, k, m} a_{j, k, m, \ell}(x), \\
f_{2}(x)=\sum_{j=0}^{\infty} \sum_{k=N}^{\infty} \sum_{m=1}^{c(j, k)} \sum_{\ell=1}^{C(j, k, m)} s_{j, k, m} a_{j, k, \ell}(x) .
\end{gathered}
$$


Hence $f=f_{1}+f_{2}$ and (36) imply

$$
\left\|f_{2} \mid B_{p_{1}, \infty}^{s_{1}}\left(\mathbb{R}^{n}\right)\right\| \leq \varepsilon
$$

Moreover, $f_{1} \in B_{p_{0}, \infty}^{s_{0}}\left(\mathbb{R}^{n}\right)$ and $\operatorname{supp} f_{1} \subset\{x:|x| \leq N+1\}=B_{N+1}$.

So, we may interpret $f_{1}$ as an element of $B_{p_{0}, \infty}^{s_{0}}\left(B_{N+2}\right)$, where the last function space is a Besov space on the ball $B_{N+2}$ defined by restriction, $c f$. [24, Chapter 5] for details. Furthermore, there exists a constant $c_{1}>0$, depending on $N$ but not on $f_{1}$ such that

$$
c_{1}\left\|f_{1}\left|B_{p_{0}, \infty}^{s_{0}}\left(\mathbb{R}^{n}\right)\|\leq\| f_{1}\right| B_{p_{0}, \infty}^{s_{0}}\left(B_{N+2}\right)\right\| \leq\left\|f_{1} \mid B_{p_{0}, \infty}^{s_{0}}\left(\mathbb{R}^{n}\right)\right\|,
$$

cf. $[24,5.2]$. Hence, the set $E_{1}=\left\{f_{1}: f \in E\right\}$ is a bounded subset in $B_{p_{0}, \infty}^{s_{0}}\left(B_{N+2}\right)$ and, by the theorem about compact embeddings of Besov spaces on bounded domains, it is precompact in $B_{p_{1}, q_{1}}^{s_{1}}\left(\mathbb{R}^{n}\right)$, cf. [8, Section 3.3]. This and (37) show that $E$ is a precompact subset of $B_{p_{1}, \infty}^{s_{1}}\left(\mathbb{R}^{n}\right)$. Thus the embedding

$$
\left.R_{H} B_{p_{0}, \infty}^{s_{0}}\left(\mathbb{R}^{n}\right)\right) \hookrightarrow R_{H} B_{p_{1}, \infty}^{s_{1}}\left(\mathbb{R}^{n}\right)
$$

is compact. To remove the dependence on the third index $q$ one can use the elementary embedding of Besov spaces, $c f$. [19].

It remains to regard the case $p_{0}<p_{1}=\infty$. The only modification we have to make consists in a replacement of (36) by

$$
\begin{aligned}
\left\|f_{2} \mid B_{\infty, \infty}^{s_{1}}\left(\mathbb{R}^{n}\right)\right\| & \leq c \sup _{j=0,1, \ldots} \sup _{k=N, 2, \ldots} \sup _{m=0, \ldots c(j, k)}\left|s_{j, k, m}\right| \\
& \leq c N^{-b / p_{0}} \sup _{j=0,1, \ldots}\left(\sum_{k=1}^{\infty} \sum_{m=0}^{c(j, k)} C(j, k, m)\left|s_{j, k, m}\right|^{p_{0}}\right)^{1 / p_{0}} \\
& <\varepsilon
\end{aligned}
$$

The remaining arguments are unchanged.

Step 3. Now we prove the necessity of the conditions i) and ii). Since

$$
R B_{p, q}^{s}\left(\mathbb{R}^{n}\right) \hookrightarrow R_{H} B_{p, q}^{s}\left(\mathbb{R}^{n}\right)
$$

therefore the necessity of ii) follows from the fact that ii) is a necessary condition for the compactness of embeddings of radial subspaces, [19].

We prove that the condition i) is also necessary. Let us assume that i) is not satisfied. Then the exists $x \in S^{n-1}$ such that the orbit $H \cdot x$ 
is finite. Let $H \cdot x=\left\{x=x_{1}, x_{2}, \ldots, x_{m}\right\}$. We may choose $\varepsilon>0$ such that $B\left(x_{i}, \varepsilon\right) \cap B\left(x_{k}, \varepsilon\right)=\varnothing$, for any $i \neq k$. Let $\varphi \in C_{o}^{\infty}\left(\mathbb{R}^{n}\right)$ with $\operatorname{supp} \varphi \subset B(x, \varepsilon)$. We define

$$
\psi(y)=\int_{H} \varphi(h \cdot y) d h .
$$

The function $\psi$ is a smooth compactly supported function with

$$
\operatorname{supp} \psi \subset \bigcup_{i=1}^{m} B\left(x_{i}, \varepsilon\right)
$$

since $H$ is a group of isometries of $\mathbb{R}^{n}$. So we can write

$$
\psi=\sum_{i=1}^{m} \varphi_{i}, \quad \varphi_{i} \in C_{o}^{\infty}\left(\mathbb{R}^{n}\right), \quad \operatorname{supp} \varphi_{i} \subset B\left(x_{i}, \varepsilon\right) .
$$

Moreover function $\psi$ is $H$-invariant and

$$
\varphi_{k}(y)=\varphi_{i}(h \cdot y), \quad \text { if } x_{i}=h \cdot x_{k} .
$$

For any $N \in \mathbb{N}$ we put

$$
\varphi_{N, i}(y)=\varphi_{i}\left(y+x_{i}-N \cdot x_{i}\right) \quad \text { and } \quad \psi_{N}=\sum_{i=1}^{m} \varphi_{N, i} .
$$

Since, any $h \in H$ is a linear transformation and it acts on the orbit $H \cdot x$ as a permutation, we get

$$
\begin{aligned}
\psi_{N}(h \cdot y) & =\sum_{i=1}^{m} \varphi_{N, i}(h \cdot y) \\
& =\sum_{i=1}^{m} \varphi_{i}\left(h \cdot\left(y+h^{-1} \cdot\left(x_{i}-N \cdot x_{i}\right)\right)\right) \\
& =\sum_{i=1}^{m} \varphi_{i}\left(h \cdot\left(y+x_{i}-N \cdot x_{i}\right)\right) \\
& =\sum_{i=1}^{m} \varphi_{i}\left(y+x_{i}-N \cdot x_{i}\right) \\
& =\psi_{N}(y)
\end{aligned}
$$


cf. (42). Thus the function $\psi_{N}$ is $H$-invariant.

The function $\varphi_{N, i}$ are $s$-atom after suitable normalization, for any $s \in$ $\mathbb{R}$. In consequence, the exists the constant $C=C(s, p, q)>0$ independent of $N$ such that

$$
\left\|\psi_{N} \mid A_{p, q}^{s}\left(\mathbb{R}^{n}\right)\right\| \sim C(s, p, q) .
$$

But, this leads to the contradiction with compactness of the embeddings since supports of $\psi_{N}$ do not intersect each other.

Corollary 1. Let $0<p, q \leq \infty$. Then the embedding

$$
R_{H} A_{p, q}^{s}\left(\mathbb{R}^{n}\right) \hookrightarrow \operatorname{vmo}\left(\mathbb{R}^{n}\right)
$$

is compact if, and only if,

$$
R_{H} A_{p, q}^{s}\left(\mathbb{R}^{n}\right) \hookrightarrow \operatorname{bmo}\left(\mathbb{R}^{n}\right)
$$

is compact if, and only if,

$$
R_{H} A_{p, q}^{s}\left(\mathbb{R}^{n}\right) \hookrightarrow C\left(\mathbb{R}^{n}\right)
$$

is compact if, and only if,

$$
R_{H} A_{p, q}^{s}\left(\mathbb{R}^{n}\right) \hookrightarrow L_{\infty}\left(\mathbb{R}^{n}\right)
$$

is compact if, and only if, $p<\infty, s-n / p>0$ and for any $x \in S^{n-1}$ the orbit $H \cdot x$ is infinite.

Corollary 2. Let $1 \leq p_{0}, p_{1} \leq \infty$ and let $m \in \mathbb{N}$. Then the embedding

$$
R_{H} W_{p_{0}}^{m}\left(\mathbb{R}^{n}\right) \hookrightarrow L_{p_{1}}\left(\mathbb{R}^{n}\right)
$$

is compact if, and only if, $p_{0}<p_{1}, m-n / p_{0}>p_{1}$ and for any $x \in S^{n-1}$ the orbit $H \cdot x$ is infinite.

Corollary 3 (Block radial symmetry). Let $n=n_{1}+\cdots+n_{m}$ and

$$
H=S O\left(n_{1}\right) \times \cdots \times S O\left(n_{m}\right) .
$$

The embedding

$$
R_{H} A_{p_{0}, q_{0}}^{s_{0}}\left(\mathbb{R}^{n}\right) \hookrightarrow R_{H} A_{p_{1}, q_{1}}^{s_{1}}\left(\mathbb{R}^{n}\right)
$$

is compact if and only if $p_{0}<p_{1}, s_{0}-n / p_{0}>s_{1}-n / p_{1}$ and $n_{i} \geq 2$ for any $i=1, \ldots, m$. 
The first and the second corollary can be proved exactly in the same way as the analogous corollaries in [19]. The third corollary is evident.

\section{Decay properties and local smoothness of $H$-inva- riant functions.}

First we deal with a generalized version of the Strauss lemma, $c f$. [22] or [16, Chapter 2]. The approach via atomic decompositions has the additional advantage that it gives some hints about the sharpness. We will assume in this section that $H \subset O(n)$ is a close subgroup and that

$$
1 \leq b=\min _{x \in S^{n-1}} \operatorname{dim} H \cdot x .
$$

We formulate the results about the behavior near infinity and near the origin separately.

Theorem 3 (Behavior near infinity). Let $0<p \leq \infty$.

Let either $s>(n-b) / p$ and $0<q \leq \infty$ or $s=(n-b) / p$ and $0<q \leq 1$. Then there exists a constant $C$ such that

$$
|f(x)| \leq C\left\|\left.f\left|B_{p, q}^{s}\left(\mathbb{R}^{n}\right) \|\right| x\right|^{-b / p}\right.
$$

holds for all $f \in R_{H} B_{p, q}^{s}\left(\mathbb{R}^{n}\right)$ and all $|x| \geq 1$.

Remark 9. The following properties of radial functions proved in [19] say something about sharpness of the above result.

1) Let $(n-1) / n<p$. Further, let either $s<(n-b) / p$ and $0<q \leq \infty$ or $s=(n-b) / p$ and $1<q \leq \infty$. Then for all $|x| \geq 1$ there exists a sequence $\left\{f_{N}\right\}_{N=1}^{\infty}$ of radial, smooth and compactly supported functions, (depending on $x$ ) such that $\left\|f_{N} \mid B_{p, q}^{s}\right\|=1$ and $\lim _{N \rightarrow \infty}\left|f_{N}(x)\right|=\infty$.

$2)$ Let $(n-1) / n<p$. Then for all triples $(s, p, q)$ there exists a positive constant $C$ such that for all $|x| \geq 1$ there exists a smooth radial and compactly supported function $f \in R_{H} B_{p, q}^{s}\left(\mathbb{R}^{n}\right),\left\|f \mid B_{p, q}^{s}\left(\mathbb{R}^{n}\right)\right\|=1$ (depending on $x$ ) such that

$$
|f(x)| \geq C|x|^{(1-n) / p} .
$$


Theorem (Behavior near the origin). Let $0<p \leq \infty$.

Let either $s>(n-b) / p$ and $0<q \leq \infty$ or $s=(n-b) / p$ and $0<q \leq 1$. Then there exists a constant $C$ such that

$$
|f(x)| \leq C\left\|\left.f\left|B_{p, q}^{s}\left(\mathbb{R}^{n}\right) \|\right| x\right|^{-b / p}\right.
$$

holds for all $f \in R B_{p, q}^{s}\left(\mathbb{R}^{n}\right)$ and all $0<|x| \leq 1$. Moreover, if $p<\infty$ we even have

$$
\lim _{|x| \rightarrow 0}|x|^{b / p} f(x)=0
$$

Remark 10. The following inverse result was proved in [19].

Let $s<1 / p$ and $0<q \leq \infty$. For all natural numbers $N$ there exists a ring with radius $\left|x_{N}\right|$ and a smooth and compactly supported radial function $f_{N}$ such that $\left\|f_{N} \mid B_{p, q}^{s}\right\|=1$ and

$$
\left|f_{N}\left(x_{N}\right)\right| \geq N\left|x_{N}\right|^{(1-n) / p} .
$$

\section{Proof of Theorems 3 and 4.}

Step 1. We comment the situation if $p=\infty$. Then all the assertions are covered by the well-known relations of $B_{\infty, q}^{s}\left(\mathbb{R}^{n}\right)$ to $C\left(\mathbb{R}^{n}\right)$, cf. [20]. The $H$-invariance does not matter. In what follows we suppose always $p<\infty$. Step 2. We prove (48) and (49).

Substep 2.1. Let $|x| \geq 1$. Let $f \in R_{H} B_{p, q}^{s}\left(\mathbb{R}^{n}\right)$. There exists an atomic decomposition

$$
f=\sum_{j=0}^{\infty} \sum_{k=0}^{\infty} \sum_{m=0}^{c(j, k)} \sum_{\ell=0}^{C(j, k, m)} s_{j, k, m} a_{j, k, m, \ell},
$$

such that (34) is satisfied. Let us fix $x \in \mathbb{R}^{n},|x| \geq 1$. Observe, that for all $j \geq 0$ there exists $k_{j} \geq 1$ such that

$$
k_{j} 2^{-j} \leq|x|<\left(k_{j}+1\right) 2^{-j} .
$$

Then the main part of $f$ near $x$ is given by the function

$$
f^{M}(y)=\sum_{j=0}^{\infty} s_{j, k_{j}, m_{j}} a_{j, k_{j}, m_{j}, \ell_{j}}(y)
$$


(in fact, $f(x)$ is a finite sum of functions of type

$$
\sum_{j=0}^{\infty} s_{j, k_{j}, m_{j}} a_{j, k_{j}+r_{j}, m_{j}+u_{j}, \ell_{j}+t_{j}}(y)
$$

and $\left|r_{j}\right|, u_{j}$ and $\left|t_{j}\right|$ are uniformly bounded). For convenience we give an estimate of the main part $f^{M}$ only. Because of (52) and the normalization of the atoms we obtain

$$
\begin{aligned}
\left|f^{M}(x)\right| & \leq \sum_{j=0}^{\infty}\left|s_{j, k_{j}, m_{j}}\right| 2^{-j(s-n / p)} \\
& \leq \sum_{j=0}^{\infty} k_{j}^{-b / p} 2^{-j(s-n / p)}\left(\sum_{k=1}^{\infty} \sum_{m=0}^{c(j, k)} k^{b}\left|s_{j, k, m}\right|^{p}\right)^{1 / p} \\
& \leq C|x|^{-b / p} \sum_{j=0}^{\infty} 2^{-j(s-(n-b) / p)}\left(\sum_{k=1}^{\infty} \sum_{m=0}^{c(j, k)} C(j, k, m)\left|s_{j, k, m}\right|^{p}\right)^{1 / p} \\
& \leq C|x|^{-b / p}\left\|f \mid B_{p, q}^{s}\left(\mathbb{R}^{n}\right)\right\|,
\end{aligned}
$$

as long as $s>(n-b) / p$ or $s=(n-b) / p$ and $0<q \leq 1$. Moreover, $C$ does not depend on $x$ and $f$.

Substep 2.2. Let $0<|x|<1$. The counterpart of (52) is given by

$$
2^{-j_{0}} \leq|x|<2^{-j_{0}+1},
$$

for some $j_{0} \geq 1$. Then the main part of $f$ near $x$ is taken by the function

$$
f^{M}(y)=\sum_{j=0}^{j_{0}-1} s_{j, 0,0} a_{j, 0,0,0}(y)+\sum_{j=j_{0}}^{\infty} s_{j, k_{j}, m_{j}} a_{j, k_{j}, m_{j}, \ell_{j}}(y) .
$$

Similar as above we derive

$$
\left|f^{M}(x)\right| \leq \sum_{j=0}^{j_{0}-1}\left|s_{j, 0,0}\right| 2^{-j(s-n / p)}+\sum_{j=j_{0}}^{\infty}\left|s_{j, k_{j}, m_{j}}\right| 2^{-j(s-n / p)} .
$$

It remains to deal with the first sum on the right-hand side. By the monotonicity of the quasi-norms with respect to $s$ and $q$ it is sufficient to concentrate on $s=(n-b) / p$ and $q=1$. This gives

$$
\begin{aligned}
\sum_{j=0}^{j_{0}-1}\left|s_{j, 0,0}\right| 2^{j b / p} & \leq|x|^{-b / p} \sum_{j=0}^{j_{0}-1} 2^{\left(1-j_{0}\right) b / p} 2^{j b / p}\left|s_{j, 0,0}\right| \\
& \leq C_{2}|x|^{-b / p}\left\|f \mid B_{p, q}^{s}\left(\mathbb{R}^{n}\right)\right\| .
\end{aligned}
$$


Now, (53) and (55) prove the claim.

Step 3. We prove (50). As in Substep 2.2 we obtain

$$
\begin{aligned}
& |x|^{b / p}\left|f^{M}(x)\right| \\
& \leq \sum_{j=0}^{j_{0}-1}\left|s_{j, 0,0}\right| 2^{\left(j-j_{0}\right) b / p}+\sum_{j=j_{0}}^{\infty}\left|s_{j, k_{j}, m_{j}}\right| 2^{\left(j-j_{0}\right) b / p} \\
& \leq\left(\sup _{j=0,1, \ldots}\left|s_{j, 0,0}\right|\right) \sum_{j=0}^{j_{0} / 2} 2^{\left(j-j_{0}\right) b / p}+\left(\sup _{j \geq j_{0} / 2}\left|s_{j, 0,0}\right|\right) \sum_{j=j_{0} / 2}^{j_{0}-1} 2^{\left(j-j_{0}\right) b / p} \\
& \quad+\sum_{j=j_{0}}^{\infty}\left(\sum_{k=1}^{\infty} k^{b}\left|s_{j, k, m}\right|^{p}\right)^{1 / p} \\
& \leq C\left(\sup _{j=0,1, \ldots}\left|s_{j, 0,0}\right|\right) 2^{-b j_{0} /(2 p)}+\left(\sup _{j \geq j_{0} / 2}\left|s_{j, 0,0}\right|\right) \\
& \left.\quad+\sum_{j=j_{0}}^{\infty}\left(\sum_{k=1}^{\infty} C(j, k, m)\left|s_{j, k, m}\right|^{p}\right)^{1 / p}\right)
\end{aligned}
$$

since $k_{j} \sim 2^{j-j_{0}}$. If $|x| \longrightarrow 0$, then $j_{0} \longrightarrow \infty$. Further, if $j_{0} \longrightarrow \infty$, then $\sum_{j \geq j_{0}}\left|s_{j, 0,0}\right| \longrightarrow 0$. Finally, also the second sum in the latter inequality tends to zero if $j_{0} \longrightarrow \infty$. From this (50) follows.

For later use we add the following observation about the convergence of partial sums of the series in (33). Let $f \in R_{H} B_{p, q}^{s}\left(\mathbb{R}^{n}\right)$ be given by

$$
f(x)=\sum_{j=0}^{\infty} \sum_{k=1}^{\infty} \sum_{m=1}^{c(j, k)} \sum_{\ell=1}^{C(j, k, m)} s_{j, k, m} a_{j, k, m, \ell}
$$

then we put

$$
S^{J} f(x)=\sum_{j=0}^{J} \sum_{k=0}^{J} \sum_{m=0}^{c(j, k)} \sum_{\ell=0}^{C(j, k, m)} s_{j, k, m} a_{j, k, m, \ell}, \quad J=0,1, \ldots
$$

Lemma 3. Let $0<p<\infty$. Suppose either $s>(n-b) / p$ and $0<q \leq \infty$ or $s=(n-b) / p$ and $0<q \leq 1$. Let $\varepsilon>0$. Then $f \in R_{H} B_{p, q}^{s}\left(\mathbb{R}^{n}\right)$ implies $f$ is continuous in $\{x:|x|>\varepsilon\}$ and

$$
\lim _{J \rightarrow \infty}\left\|f-S^{J} f \mid L_{\infty}(\{x:|x|>\varepsilon\})\right\|=0 .
$$


Proof. We employ the same arguments as in proof of Theorems 3 and 4, Step 2. Thanks to $f \in R_{H} B_{p, q}^{s}\left(\mathbb{R}^{n}\right)$ for each $\delta>0$ there exists a number $J_{0}$ such that

$$
\left(\sum_{j=J}^{\infty}\left(\sum_{k=0}^{\infty} \sum_{m=0}^{c(j, k)} C(j, k, m)\left|s_{j, k, m}\right|^{p}\right)^{q / p}\right)^{1 / q}<\delta,
$$

for all $J \geq J_{0}$. First, consider $|x| \geq 1$. Then, as in (53)

$$
\left|f^{M}(x)-S^{J} f^{M}(x)\right|=\left|\sum_{j=J+1}^{\infty} s_{j, k_{j}, m_{j}} a_{j, k_{j}, m_{j}, \ell_{j}}(x)\right| \leq C|x|^{-b / p} \delta,
$$

for all $J \geq J_{0}$, with $C$ independent of $x$ and $\delta$. Similar, if $\max \left\{\varepsilon, 2^{-j_{0}}\right\} \leq$ $|x|<2^{-j_{0}+1}$ and $J$ is sufficiently large with respect to $\varepsilon$, then also in this case we arrive at (56). Hence, we have convergence of $S^{J} f$ in the uniform norm and for that reason the limit itself is a continuous function. This proves the lemma.

Next we investigate local Hölder regularity of $\mathrm{H}$-invariant functions. Let $\psi$ be a radial smooth cut-off function supported around the origin and satisfying $\psi(x)=1$ if $|x| \leq 1$.

Any $x \in S^{n-1}$ defines a ray $\rho_{x}=\left\{y \in \mathbb{R}^{n}: y=t x, t>0\right\}$. To a function $f: \mathbb{R}^{n} \longrightarrow \mathbb{C}$ we associate a function $f_{x}:[0, \infty) \longrightarrow \mathbb{C}$ by $f_{x}(t)=f(t x), x \in S^{n-1}$. We say that a function $f_{x}$ belongs to $B_{\infty, \infty}^{s}(0, \infty)$ if the functions $(1-\psi(\lambda|y|)) f_{0}(|y|)$ belong to $B_{\infty, \infty}^{s}(\mathbb{R})$ for all $\lambda>0$.

Lemma 4. Let $0<p, q \leq \infty$. Suppose $s>(n-b) / p$. Let $f \in R_{H} B_{p, q}^{s}\left(\mathbb{R}^{n}\right)$. Then for any $x \in S^{n-1}, f_{x}$ belongs to $B_{\infty, \infty}^{s_{o}}(0, \infty)$, with $s_{o}=s-(n-b) / p$.

\section{Proof.}

Step 1. First, we remark that the case $p=\infty$ is obvious by employing the equivalence of the quasi-norms $\left\|f \mid B_{\infty, \infty}^{s}\left(\mathbb{R}^{n}\right)\right\|$ and

$$
\sup _{x \in \mathbb{R}^{n}}|f(x)|+\max _{|\alpha| \leq m} \sup _{\substack{x, y \in \mathbb{R}^{n} \\ x \neq y}} \frac{\left|D^{\alpha} f(x)-D^{\alpha} f(y)\right|}{|x-y|^{s-m-1 / p}}, \quad m<s-\frac{n-b}{p}<m+1,
$$

or

$$
\sup _{x \in \mathbb{R}^{n}}|f(x)|+\max _{|\alpha| \leq m} \sup _{\substack{x, h \in \mathbb{R}^{n} \\ h \neq 0}} \frac{\left|D^{\alpha} f(x+2 h)-2 D^{\alpha} f(x+h)+D^{\alpha} f(x)\right|}{|h|},
$$


$s-(n-b) / p=m+1$.

Step 2. Let $p<\infty$. Let $f \in R_{H} B_{p, q}^{s}\left(\mathbb{R}^{n}\right)$ for some $s>(n-b) / p$. The function $(1-\psi(\lambda x))$ is a pointwise multiplier for $B_{p, q}^{s}\left(\mathbb{R}^{n}\right)$, cf. e.g. $[23,2.8]$ or [10], and hence, also $(1-\psi(\lambda x)) f(x)$ belongs to $R B_{p, q}^{s}\left(\mathbb{R}^{n}\right)$. Lemma 3 yields that it is a continuous function. Moreover there are constants $N$ and $K$ dependent on $\lambda$ such that the function $(1-\psi(\lambda \cdot)) f$ has the following atomic decomposition

$$
\begin{aligned}
& (1-\psi(\lambda x)) f(x) \\
& =\sum_{j=0}^{N} s_{j, 0,0} a_{j, 0,0,0}+\sum_{j=0}^{\infty} \sum_{k \geq \max \left\{1,2^{j} K\right\}} \sum_{m=1}^{c(j, k)} \sum_{\ell=1}^{C(j, k, m)} s_{j, k, m} a_{j, k, m, \ell},
\end{aligned}
$$

cf. (33). To simplify the notation we may assume that $x=(1,0, \ldots, 0)$ and restrict our attention to the $x_{1}$-axis. We have

$$
\begin{aligned}
&\left(1-\psi\left(\lambda\left(x_{1}, 0, \ldots, 0\right)\right)\right) f\left(x_{1}, 0, \ldots, 0\right) \\
&=\sum_{j=0}^{N} s_{j, 0,0} a_{j, 0,0,0}\left(x_{1}, 0, \ldots, 0\right) \\
&+\sum_{j=0}^{\infty} \sum_{k \geq \max \left\{1,2^{j} K\right\}} \sum_{m, \ell} s_{j, k, m} a_{j, k, m, \ell}\left(x_{1}, 0, \ldots, 0\right),
\end{aligned}
$$

(counting the $a_{j, k, m, \ell}$ in an appropriate way). The functions $b_{j, k, m, \ell}\left(x_{1}\right)=$ $2^{-j b / p} a_{j, k, m, \ell}\left(x_{1}, 0, \ldots, 0\right)$ are $(s+1 / p-(n-b) / p, p)_{L,-1}$-atoms on $x_{1}$-axis. It follows that

$$
g_{m, \ell}=\sum_{j=0}^{N} 2^{j b / p} s_{j, 0,0} b_{j, 0,0,0}+\sum_{j=0}^{\infty} \sum_{k \geq \max \left\{1,2^{j} K\right\}} 2^{j b / p} s_{j, k, m} b_{j, k, m, \ell},
$$

$\ell=1, \ldots, M$, represent tempered distributions on $\mathbb{R}$ satisfying

$$
\begin{aligned}
& \left\|g_{m, \ell} \mid B_{p, \infty}^{\sigma}(\mathbb{R})\right\| \\
& \leq C\left(\sup _{j=0, \ldots, N} 2^{j b / p}\left|s_{j, 0,0}\right|+\sup _{j=0, \ldots}\left(\sum_{k \geq \max \left\{1,2^{j} K\right\}}\left|2^{j b / p} s_{j, k, m}\right|^{p}\right)^{1 / p}\right) \\
& \leq C\left(\sup _{j=0, \ldots, N}\left|s_{j, 0,0,0}\right|+\sup _{j=0, \ldots}\left(\sum_{k=1}^{\infty} \sum_{m} C(j, k, m)\left|s_{j, k, m}\right|^{p}\right)^{1 / p}\right) \\
& \leq C\left\|f \mid B_{p, \infty}^{s}\left(\mathbb{R}^{n}\right)\right\|,
\end{aligned}
$$


with $\sigma=s+1 / p-(n-b) / p$ and $C$ does not depend on $f$.

Step 3. The trace problem. Consider the mapping

$$
\gamma_{L}: f\left(x_{1}, x_{2}, \ldots, x_{n}\right) \longmapsto\left(1-\psi\left(2^{-L}\left(x_{1}, 0, \ldots, 0\right)\right) f\left(x_{1}, 0, \ldots, 0\right),\right.
$$

$f \in C\left(\mathbb{R}^{n}\right)$. Let $S^{J} f$ be the partial sum of the atomic decomposition from Lemma 3. Because of $s>1 / p$ there exists a number $s_{0}$ such that $1 / p<s_{0}<s$ and $f \in R B_{p, 1}^{s_{0}}\left(\mathbb{R}^{n}\right)$. Moreover

$$
S^{J} f(x) \longrightarrow f
$$

in $\left\|\cdot \mid B_{p, 1}^{s_{0}}\left(\mathbb{R}^{n}\right)\right\|$ if $J \longrightarrow \infty$. Because of

$$
\begin{aligned}
& \gamma_{L}\left(S^{J} f\right) \\
& =\sum_{m, \ell} \sum_{j=0}^{\min \{J, N\}} 2^{j b / p} s_{j, 0,0} b_{j, 0,0,0}+\sum_{j=N}^{J} \sum_{k \geq \max \left\{1,2^{j} K\right\}} 2^{j b / p} s_{j, k, m} b_{j, k, m, \ell} .
\end{aligned}
$$

Step 2 implies the convergence of $\gamma_{L}\left(S^{J} f\right)$ in $\|\cdot \mid C(\mathbb{R})\|$ (thanks to the continuous embedding $\left.B_{p, \infty}^{s}(\mathbb{R}) \hookrightarrow C(\mathbb{R})\right)$. Consequently, $\gamma_{L}$ extends to a bounded linear mapping of $R B_{p, q}^{s}\left(\mathbb{R}^{n}\right)$ into $R B_{p, q}^{s}(\mathbb{R})$.

Lemma 3 implies coincidence (pointwise) of $\gamma_{L}(f)$ and $\left(1-\psi\left(2^{-L}|y|\right)\right)$ $\cdot f_{x}(|y|)$. The prove is complete.

Remark 11. The phenomenon of the higher local regularity of radial functions outside the origin has been observed by P. L. Lions [17].

We have proved more than stated. Namely for any $f$ with $\operatorname{supp} F \subset$ $\mathbb{R}^{n} \backslash B(0, R)$ the following inequality

$$
\left\|f_{0}\left|B_{\infty, q}^{s_{o}}(\mathbb{R})\|\leq\| f_{0}\right| B_{p, q}^{\sigma}(\mathbb{R})\right\| \leq C R^{-b / p}\left\|f \mid B_{p, q}^{s}\left(\mathbb{R}^{n}\right)\right\|
$$

holds with the constant $C$ independent of $f$ and $\lambda$. In fact, since in (57) we have $\left(R 2^{j}\right)^{b / p} \leq C(j, k, m)$ therefore for $q=\infty$ the inequality (58) follows from (57). The extension does not cause any trouble.

Lemma 5. Let $H \subset O(n)$ be a compact subgroup. We assume that $H$ do not acts transitively on $S^{n-1}$ and that

$$
1 \leq b=\inf _{x \in S^{n-1}} \operatorname{dim} H \cdot x
$$


Let $0<p \leq \infty$. We assume that $s>(n-b) / p$ and $0<q \leq \infty$. Then for any $f \in R_{H} B_{p, q}^{s}\left(\mathbb{R}^{n}\right)$ the exists a trace $\tilde{f}$ of $f$ on $S_{R}^{n-1}$ and

$$
\widetilde{f} \in B_{p_{1}, q}^{\widetilde{s}}\left(S^{n-1}\right)
$$

with $p \leq p_{1} \leq \infty$ and $\widetilde{s}=s-1 / p_{1}-(n-b)\left(1 / p-1 / p_{1}\right)$. Moreover,

$$
\left\|\widetilde{f}\left|B_{p_{1}, q}^{\widetilde{s}}\left(S^{n-1}\right)\|\leq C\| f\right| B_{p, q}^{s}\left(S^{n-1}\right)\right\|
$$

where $\sigma=b\left(1 / p-1 / p_{1}\right)$.

Remark 12. 1) If $p_{1}=p$ then $\widetilde{s}=s-1 / p$. So we get the usual trace smoothness.

2) If $p<p_{1}$ then $\widetilde{s}>s-1 / p$. In particular if $p_{1}=\infty$ then $\widetilde{s}=s-$ $(n-b) / p$. So the function $\tilde{f}$ is Hölder continuous on $S^{n-1}$ with smoothness coefficient $s-(n-b) / p>0$.

Proof. The sphere $S^{n-1}$ is a compact manifold so we can use the atomic decomposition of the functions spaces on manifolds with bounded geometry. We refer to [21] for details. The function $f$ is continuous since $s>(n-b) / p, c f$. Lemma 3. Once more we regard only the main part of $f$ near $S^{n-1}$. It is given by the function

$$
f^{M}(y)=\sum_{j=0}^{\infty} \sum_{m=0}^{C\left(j, k_{j}\right)} \sum_{\ell=0}^{C\left(j, k_{j}, m\right)} s_{j, k_{j}, m} a_{j, k_{j}, m, \ell}(y)
$$

where $2^{-j} k_{j} \sim 1$. In fact, $f(x)$ is a finite sum of functions of the above type.

We note that there is a constant $C>0$

$$
C\left(j, k_{j}, m\right) \geq C 2^{j b}
$$

$c f .(25)$. Let $\sigma=b\left(1 / p-1 / p_{1}\right)$. We put

$$
\begin{gathered}
\widetilde{a}_{j, k_{j}, m, \ell}=\left.2^{-j \sigma} a_{j, k_{j}, m, \ell}\right|_{S^{n-1}}, \\
\widetilde{s}_{j, k_{j}, m}=2^{j \sigma} s_{j, k_{j}, m} .
\end{gathered}
$$

The above functions are $\left(\widetilde{s}, p_{1}\right)$-atoms on $S^{n-1}$. Since, $\widetilde{s}>0$ no moment for atoms is needed, $c f$. [21]. 
Let

$$
\widetilde{f^{M}}(y)=\sum_{j=0}^{\infty} \sum_{m=0}^{C\left(j, k_{j}\right)} \sum_{\ell=0}^{C\left(j, k_{j}, m\right)} \widetilde{s}_{j, k_{j}, m} \widetilde{a}_{j, k_{j}, m, \ell}(y)
$$

The last decomposition is an atomic decomposition of $\widetilde{f^{M}}$ on $S^{n-1}$, since

$$
\begin{aligned}
\left(\sum_{j=0}^{\infty}\left(\sum_{m=0}^{C\left(j, k_{j}\right)} C\left(j, k_{j}, m\right)\left|\widetilde{s}_{j, k_{j}, m}\right|^{p_{1}}\right)^{q / p_{1}}\right)^{1 / q} \\
\leq\left(\sum_{j=0}^{\infty}\left(\sum_{m=0}^{C\left(j, k_{j}\right)} C\left(j, k_{j}, m\right)\left|s_{j, k_{j}, m}\right|^{p}\right)^{q / p}\right)^{1 / q}<\infty
\end{aligned}
$$

But the last inequalities and the atomic decomposition theorem imply

$$
\left\|\widetilde{f^{M}}\left|B_{p_{1}, q}^{\widetilde{s}}\left(S^{n-1}\right)\|\leq C\| f^{M}\right| B_{p, q}^{s}\left(\mathbb{R}^{n}\right)\right\| .
$$

It follows from Lemma 3 that $\widetilde{f^{M}}$ is a trace of $f^{M}$ on $S^{n-1}$. This finishes the proof.

The last two lemmas give us the following theorem.

Theorem 5. Let $H \subset O(n)$ be a compact subgroup and $b=\inf _{x \in S^{n-1}}$ $\operatorname{dim} H \cdot x$. Let $0<p \leq \infty$ and $0<q \leq \infty$. We assume that $s>$ $(n-b) / p$. Let $\psi$ be a radial smooth cut-off function supported around the origin and satisfying $\psi(x)=1$ if $|x| \leq 1$. Then for any $\lambda>0$ and any $f \in$ $R_{H} B_{p, q}^{s}\left(\mathbb{R}^{n}\right)$ the function $(1-\psi(\lambda \cdot)) f \in B_{\infty, \infty}^{s_{o}}\left(\mathbb{R}^{n}\right)$, with $s_{o}=s-(n-b) / p$. Moreover, the operator $R_{H} B_{p, q}^{s}\left(\mathbb{R}^{n}\right) \ni f \longmapsto(1-\psi(\lambda \cdot)) f \in B_{\infty, \infty}^{s_{o}}\left(\mathbb{R}^{n}\right)$ is bounded.

\section{Proof.}

Step 1 . Let us choose a point $x \in \mathbb{R}^{n}$ with $|x|=\lambda^{-1}$. Let $x=\left(\lambda^{-1}, \theta_{o}\right)$ be the polar coordinates of $x$. For $0<\varepsilon<\lambda^{-1} / 2$ we defined the neighborhood $U_{\varepsilon}$ of $x$ by

$$
U_{\varepsilon}=\left\{(r, \theta):\left|r-\lambda^{-1}\right|<\varepsilon,\left|\theta-\theta_{o}\right|<\varepsilon\right\}
$$

Let $\varphi \in C_{0}^{\infty}\left(\mathbb{R}^{n}\right)$ be a smooth compactly supported function such that $\operatorname{supp} \varphi \subset B(x, \delta) \subset U_{\varepsilon}, 0<\delta$.

Let $\Psi$ be a diffeomorphism of $U_{\varepsilon}$ onto $(0,1)^{n}$ such that: 

axis,

- the lines $r \longrightarrow(r, \theta)$ are mapped onto the lines parallel to the $x_{1^{-}}$

- the surfaces of the spheres centered at the origin are mapped to the hyperplanes orthogonal to the $x_{1}$-axis.

If a function $f$ is supported in $U_{\varepsilon}$ than by the diffeomorphism properties of the Besov spaces a function $f \in B_{\infty, \infty}^{s}\left(\mathbb{R}^{n}\right)$ if and only if $\widetilde{f}=$ $f \circ \Psi^{-1} \in B_{\infty, \infty}^{s}\left(\mathbb{R}^{n}\right), c f$. [23, Theorem 2.10.2]. Lemma 4 implies that for any $\widetilde{x} \in(0,1)^{n-1}$ the function $\widetilde{f}(\cdot, \widetilde{x})$ is an element of $B_{\infty, \infty}^{s_{o}}(\mathbb{R})$ and

$$
\left\|\tilde{f}(\cdot, \widetilde{x})\left|B_{\infty, \infty}^{s_{o}}(\mathbb{R})\|\leq C\| f\right| B_{p, q}^{s}\left(\mathbb{R}^{n}\right)\right\|
$$

Here the constant $C$ depend on $\lambda$ but not $\widetilde{x}$. In the similar way, Lemma 5 and (61) implies that for any $x_{1} \in(0,1)$ the function $\widetilde{f}\left(x_{1}, \cdot\right)$ is an element of $B_{\infty, \infty}^{s_{o}}\left(\mathbb{R}^{n-1}\right)$ and $B_{\infty, \infty}^{s_{o}}(\mathbb{R})$ and

$$
\left\|\tilde{f}\left(x_{1}, \cdot\right)\left|B_{\infty, \infty}^{s_{o}}\left(\mathbb{R}^{n-1}\right)\|\leq C\| f\right| B_{p, q}^{s}\left(\mathbb{R}^{n}\right)\right\| .
$$

Now the Fubini type theorem for Besov spaces implies that $\tilde{f} \in B_{\infty, \infty}^{s_{o}}\left(\mathbb{R}^{n}\right)$, cf. [23, Theorem 2.5.13]. Thus $f \in B_{\infty, \infty}^{s_{o}}\left(\mathbb{R}^{n}\right)$ and

$$
\left\|f\left|B_{\infty, \infty}^{s_{o}}\left(\mathbb{R}^{n}\right)\|\leq C\| f\right| B_{p, q}^{s}\left(\mathbb{R}^{n}\right)\right\| .
$$

By rotations the last inequality holds for any choosen $x,|x|=\lambda^{-1}$, with the same constant $C$.

Step 2. If the function $f$ is supported in the set $U_{\varepsilon, R}=\left\{x \in \mathbb{R}^{n}: x=\right.$ $\left.R y, y \in U_{\varepsilon}\right\}$ then the usual scaling argument with dilations, $c f$. [8, Theorem 2.3.1], implies

$$
\begin{aligned}
\left\|f \mid B_{\infty, \infty}^{s_{o}}\left(\mathbb{R}^{n}\right)\right\| & \leq C R^{-s_{o}}\left\|f(R \cdot) \mid B_{\infty, \infty}^{s_{o}}\left(\mathbb{R}^{n}\right)\right\| \\
& \leq C R^{-s_{o}}\left\|f(R \cdot) \mid B_{p, q}^{s}\left(\mathbb{R}^{n}\right)\right\| \\
& \leq C R^{s-s_{o}-n / p}\left\|f \mid B_{p, q}^{s}\left(\mathbb{R}^{n}\right)\right\| \\
& =C R^{-b / p}\left\|f \mid B_{p, q}^{s}\left(\mathbb{R}^{n}\right)\right\|,
\end{aligned}
$$

where $C$ is a constant depending on $\lambda$ but independent of $R$ and $f$.

We can cover the anulus $B\left(0,3 \lambda^{-1} / 2\right) \backslash B\left(0, \lambda^{-1} / 2\right)$ by the finite family of the set of the type (59) and then we can cover $\mathbb{R}^{n} \backslash B\left(0, \lambda^{-1} / 2\right)$ by the dilatations of these sets. One can find also a resolution of unity coresponding to the above covering with the following property: if a function 
$\phi$ is supported in $U_{\varepsilon, R}$ then $\left|\partial^{\alpha} \phi(R \cdot)\right| \leq C_{\alpha}$ for any multi-index $\alpha$. Now the theorem follows by the localization property for the space $B_{\infty, \infty}^{s_{o}}\left(\mathbb{R}^{n}\right)$.

Remark 13. Since we can control the constants in the last proof we can stated even more. Namely the following inequality

$$
\left\|f\left|B_{\infty, \infty}^{s_{o}}\left(\mathbb{R}^{n}\right)\left\|\leq C R^{-b / p}\right\| f\right| B_{p, q}^{s}\left(\mathbb{R}^{n}\right)\right\|
$$

holds for any $H$-invariant function $f$ with supp $f \subset \mathbb{R}^{n} \backslash B(0, R), R>0$. The constant $C$ is independent of $f$ and $R$.

\section{References.}

[1] Berestycki, B., Lions, P.-L., Existence of a ground state in nonlinear equations of the type Klein-Gordon. In: Variational inequalities and complementary theory and applications. Wiley, 1979.

[2] Bredon, G. L., Introduction to compact transformation groups. Academic Press, 1972.

[3] Chavel, I., Riemannian Geometry Modern Introduction. Cambridge Univ. Press, 1994.

[4] Cheeger, J., EBin, D. G., Comparison Theorems in Riemannian Geometry. North-Holland, 1975.

[5] Coleman, S., Glazer, V., Martin, A., Action minima among solutions to a class of euclidean scalar field equations. Comm. Math. Phys. 58 (1978), 211-221.

[6] Ding Yi, Non-radial solutions of semilinear elliptic equations. Math. Acta Sci. 10 (1990), 229-239.

[7] Ebihara, Y., Schonbek, T. P., On the (non)compactness of the radial Sobolev spaces. Hiroshima Math. J. 16 (1986), 665-669.

[8] Edmunds, D. E., Triebel, H., Function spaces, entropy numbers and differential operators. Cambridge Univ. Press, 1966.

[9] Frazier, M., Jawerth, B., Decomposition of Besov spaces. Indiana Univ. Math. J. 34 (1985), 777-799.

[10] Frazier, M., JaWerth, B., A discrete transform and decomposition of distribution spaces. J. Funct. Anal. 93 (1990), 34-170.

[11] Hebey, E., Vaugon, M., Sobolev spaces in the presence of symmetries. J. Math. Pures Appl. 76 (1997), 859-881.

[12] Helgason, S., Differential Geometry, Lie Groups and Symmetric Spaces. Academic Press, 1978.

[13] Helgason, S., Geometric Analysis on Symmetric Spaces. Math. Surveys and Monographs. A. M. S., 1994. 
[14] Jawerth, B., Some observations on Besov and Lizorkin-Triebel spaces. Math. Scan. 40 (1977), 94-104.

[15] Kuzin, I., Existence theorems for nonlinear elliptic problems in $\mathbb{R}^{N}$ in the class of block-radial functions. Differential Equations 30 (1994), 637-646.

[16] Kuzin, I., Pohozaev, S., Entire solutions of semilinear elliptic equations. Birkhäuser, 1997.

[17] Lions, P.-L., Symétrie et compacité dans les espaces de Sobolev. J. Funct. Anal. 49 (1982), 315-334.

[18] Peetre, J., New Thoughts on Besov spaces. Duke Univ. Math. Series. Duke Univ., 1976.

[19] Sickel, W., Skrzypczak, L., Radial subspaces of Besov and LizorkinTriebel classes: extended Strauss lemma and compactness of embedding. $J$. Fourier Analysis Appl. 6 (2000), 639-662.

[20] Sickel, W., Triebel, H., Hölder inequality and sharp embeddings in function spaces of $B_{p q}^{s}$ and $F_{p q}^{s}$ type. Z. Anal. Anwendungen 14 (1995), 105-140.

[21] Skrzypczak, L., Atomic decompositions on manifolds with bounded geometry. Forum Math. 10 (1998), 19-38.

[22] Strauss, W. A., Existence of solitary waves in higher dimensions. Comm. Math. Phys. 55 (1977), 149-162.

[23] Triebel, H., Theory of Function Spaces. Birkhäuser, 1983.

[24] Triebel, H., Theory of Function Spaces II. Birkhäuser, 1992.

Recibido: 15 de febrero de 2000

Revisado: 30 de abril de 2001

Leszek Skrzypczak

Faculty of Mathematics and Computer Science

A. Mickiewicz University

Matejki 48-49

60-769 Poznań, POLAND

lskrzyp@amu .edu.pl 